\title{
Ultrananocrystalline Diamond Integration with Pyrolytic Carbon Components of Mechanical Heart Valves
}

\author{
$\underline{\text { Hongjun Zeng }}{ }^{1}$, Wei Yin ${ }^{2}$, Grace Catausan ${ }^{1}$, Nicolaie Moldovan ${ }^{1}$, and John Carlisle ${ }^{1}$ \\ ${ }^{1}$ Advanced Diamond Technologies, Inc. 48 E Belmont Drive, Romeoville, IL 60446, USA \\ zeng@thindiamond.com \\ ${ }^{2}$ Department of Biomedical Engineering, Stony Brook University, Stony Brook, NY 11794
}

\begin{abstract}
In this report, an idea of integrating ultrananocrystalline diamond (UNCD) with pyrolytic carbon (PyC) based mechanical heart valves, has been demonstrated. The report addresses the strategies to avoid graphitization and film delamination during the diamond coating. Raman and scratch tests showed that a UNCD film with high purity could adhere to the PyC substrate strongly. A thrombin generation study demonstrated an excellent biocompatibility of UNCD towards fresh human platelets. These results suggest that UNCD could be a good candidate of surface material for next generation heart valves and other implantable devices.
\end{abstract}

Key words: Ultrananocrystalline diamond (UNCD), pyrolytic carbon, mechanical heart valve, biocompatibility, implantable devices

\section{Introduction}

Pyrolytic carbon (PyC) is the most widely used structural material for mechanical heart valves due to its biocompatibility, low cost and ease of manufacturing [1]. However, PyC components still fails and releases carbon particles into blood due to scratches at valve hinge area, which may cause blood contamination [2-3]. At the same time, although $\mathrm{PyC}$ has better biocompatibility than many other materials currently employed for medical purposes, patients who receive mechanical heart valves still need to take anticoagulation drugs for the rest of their life [4]. As an alternative, tissue valves eliminate the usage of anticoagulants, but the lifespan of tissue valves is usually less than 15 years and the patients may need to go through multiple thoracic surgeries. Therefore, a better material may help to optimize the mechanical heart valve design and lead to the next generation of heart valves, with both longer lifetime and less anticoagulant dependence. UNCD has been reported to have exceptional chemical inertness, biocompatibility, especially mechanical durability and tribological properties due to its diamond nature and unmatched smoothness [5-6]. The idea of this report is to integrate a thin layer (a few microns) of UNCD onto PyC heart valves, to enhance the performances of the heart valves, while not sacrificing PyC's original advantages of low cost and ease of manufacturing.

Like graphite, PyC tends to absorb carbon species if it is loaded in a chemical vapor deposition (CVD) reactor for diamond deposition. As a result, the regular UNCD deposition process is seriously prone to 
produce graphite which affects the film purity and performance. At the same time, the generated graphite is porous, such that even if some diamond crystallites are eventually growing, they cannot anchor on a solid substrate. Therefore, the film's adhesion on PyC is usually poor. With the intrinsic stress, diamond film usually delaminates from the substrate during or soon after the deposition. Due to such a technical challenge, successful diamond coating on $\mathrm{PyC}$, pyrolytic graphite or other graphite forms was rarely reported. In this work, we minimized the graphitization during the deposition and improved the diamond adhesion with a few methods including introducing an interlayer, stress matching and carbide bonding between the film and the PyC substrate.

\section{Experimental procedures}

The pyrolytic carbon coupons, leaflets and orifices of heart valves were used for the diamond deposition. Manufacturing of PyC has been well developed [7]. The PyC substrates were firstly coated with a thin, 20-100 nm tungsten via either sputtering or atomic layer deposition (ALD). ALD was preferred because of its extremely conformal coating on a complicated 3-D structure. A seeding process was thereafter conducted to have diamond seeds attached on the surface of the substrates as initial nucleation centers. The seeding was achieved by commonly-accepted processes, either with surface mechanical abrasion with diamond particles or with sonication in seeding slurries containing diamond nanoparticles [8]. A hot filament chemical vapor deposition (HFCVD) reactor was employed for the UNCD coating. The setup and gas chemistry of the UNCD deposition was reported previously [9]. In the present study, we employed a similar process to simulate PyC deposition at approximately $800^{\circ} \mathrm{C}$ in a $\mathrm{CH}_{4}-\mathrm{H}_{2}$ environment within a HFCVD reactor, rather than within a plasma enhanced reactor with $\mathrm{C}_{3} \mathrm{H}_{6}-\mathrm{N}_{2}$ or only $\mathrm{CH}_{4}$ [10-11]. This process consumed part of the carbon radicals at the beginning of the deposition to form a solid, PyC-like substance to gradually continue the $\mathrm{PyC}$ substrate, while preventing $\mathrm{PyC}$ from producing porous graphite. After this transit step, the gas ratio was gradually adjusted to match regular UNCD growth conditions. The overall thickness of the UNCD film was $1-2 \mu \mathrm{m}$.

A high resolution SEM was used to characterize the UNCD grains and surface morphology. The accelerating voltage, aperture size and working distance of the SEM were $10 \mathrm{kV}, 10 \mu \mathrm{m}$ and $10 \mathrm{~mm}$, respectively. Optical profilometry (Wyko NT1100) was used to measure the surface roughness of the samples before and after diamond deposition. Raman spectra were obtained using a spectrometer (Control Development 2DMPP) with an frequency-doubled neodymium-doped yttrium aluminum garnet (Nd:YAG) laser. The laser wavelength and power were $532 \mathrm{~nm}$ and $5 \mathrm{~mW}$, respectively. The film stress was measured by a Flexus Stress Measure System (Tencor FLX-2320) on Si witness wafers run in parallel with the PyC samples, with input of thicknesses of the diamond film and the parameters of the substrate. The film adhesion was characterized via a Nanovea Scratch Test station and a Rockwell Ball Indenter with a Thomson Precision Ball (ceramic, 1/16"), following ASTM C1624-05, i.e. "Standard Test Method for Adhesion Strength and Mechanical Failure Modes of Ceramic Coatings by Quantitative Single Point Scratch Testing". To evaluate the biocompatibility of diamond coated components towards platelets, fresh human platelets were incubated with diamond samples for 1 hour. 
Thrombin generation was measured using a modified prothrombinase assay [12]. Calcium Ionophore A23187 was used to induce maximum platelet activation and thrombin generation, as a positive control. Untreated platelets were used as the negative control. The arithmetic average from experiments $(n=9)$ was used as a measure of thrombin generation rate induced by different surfaces. Statistical analysis was conducted by

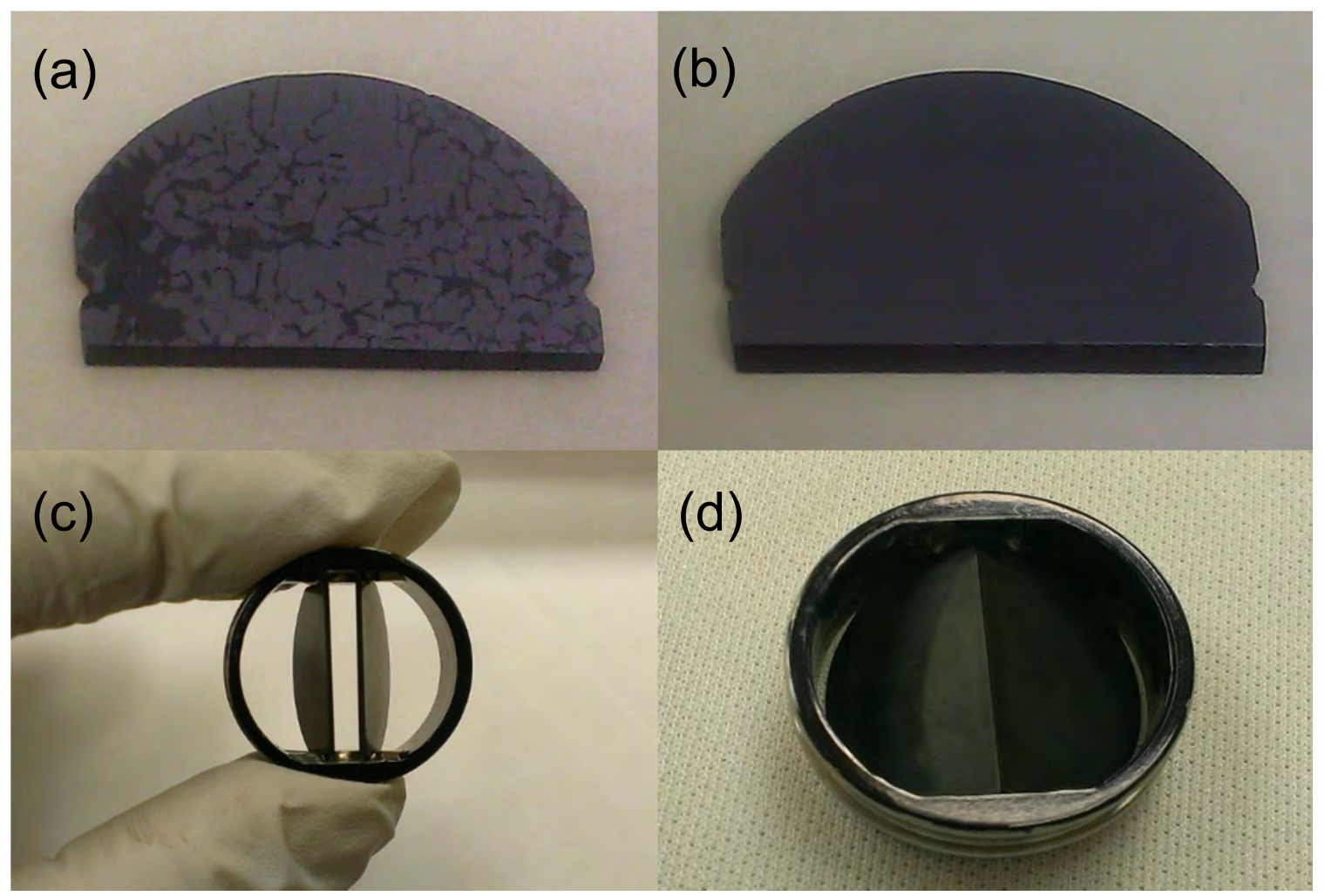

Fig 1. Leaflets that a) covered by delaminated diamond film coated with conventional technique and b) covered by solidly integrated diamond film with developed integration technique, c) are assembled into a mechanical heart valve with open status and d) with close status.

ANOVA using Microsoft EXCEL, and significant difference was set as $P=0.05$.

\section{Results and discussion}

By adding tungsten interlayer before UNCD deposition and a transit step at beginning of UNCD deposition as described above, we were able to integrate UNCD with PyC components. Figure 1a shows that when we used conventional deposition process, the diamond film catastrophically delaminated, while when we added these adhesion promoting steps (W interlayer and gradual PyC-to-UNCD transitioning), the UNCD film adhesion on the PyC substrates was significantly enhanced (Figure 1b). Figure 1c and 1d demonstrate the UNCD-coated PyC components were assembled into a mechanical heart valve. Film measurements indicated that the UNCD thickness was between 1.5-2 $\mu \mathrm{m}$. The optical profilometry measurement showed that before and after UNCD coating, the surface roughness maintained almost the same, between 50-80 nm (root mean square roughness) in a $237 \mu \mathrm{m} \times 178 \mu \mathrm{m}$ field of view. UNCD is well known for being the smoothest as-deposit surface in the diamond family, with a typical roughness of 5-8 $\mathrm{nm}$ (root mean square) [9]. Figure 
$4 \mathrm{~d}$ and $4 \mathrm{e}$ depict that the grains and grain clusters of UNCD are much smaller than the feature size of the PyC microstructure, therefore coating UNCD does not essentially add any more roughness to the substrate.

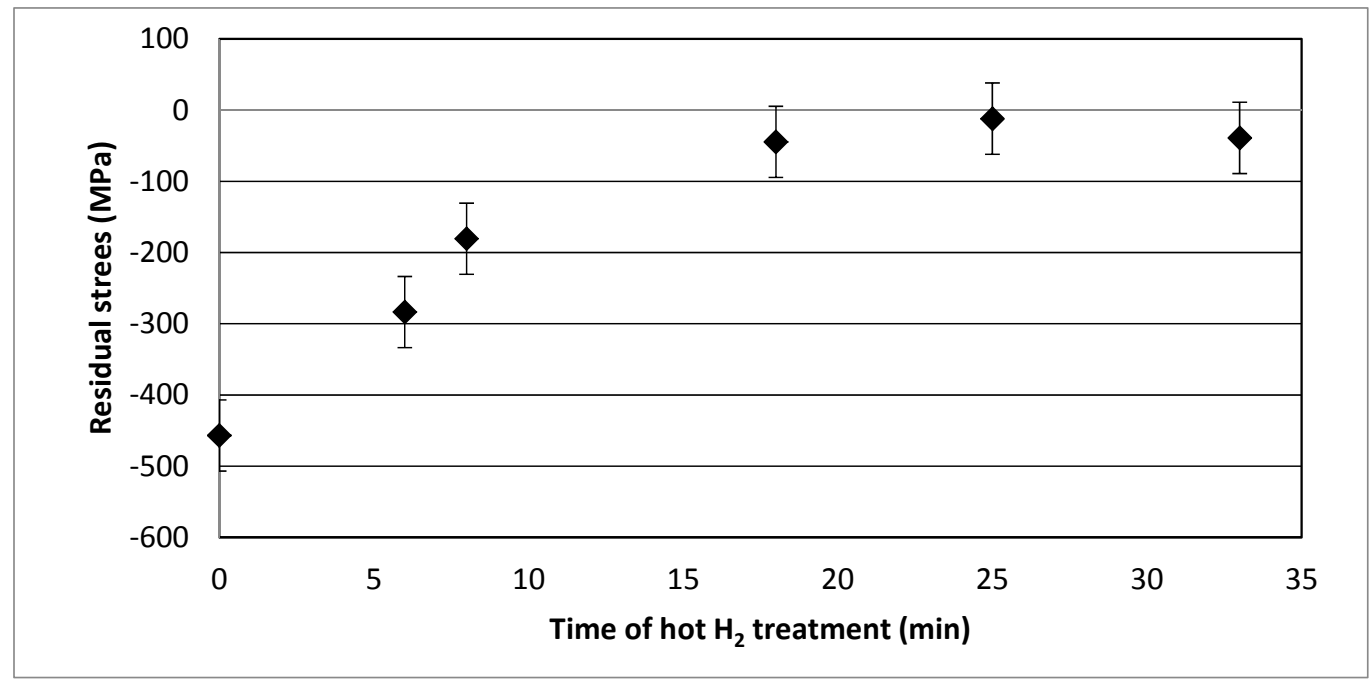

Fig 2. Residual stress vs. time of hot $\mathrm{H}_{2}$ treatment. The film thickness is $1 \mu \mathrm{m}$ on average. The stress was measured by a Tencor Flexus on 4" Si witness wafers with 525 micron thickness.

Film stress is another factor that directly affects film adhesion and shape distortion of the coated samples. It is well know that diamond film stress decreases as the deposition temperature increases. For a deposition temperature increasing from $600{ }^{\circ} \mathrm{C}$ to $850{ }^{\circ} \mathrm{C}$, the film stress can drop from - $600 \mathrm{MPa}$ (- represents compressive) to $\pm 100 \mathrm{MPa}$ ( + represents tensile), i.e. nearly stress free. Straight forward methods to raise temperature of the substrate include increasing the power of the reactor, or elevating the substrates closer to the filament. However, these methods are not practical for mass production, as raising power would interfere with the carburization balance of the filaments, which could cause tungsten filament breakage or releases tungsten carbide dust that contaminates the deposited film. Elevating the substrate closer to the filament would not only risk uneven surface heating especially for 3-D geometries, but also cause significant non-uniformity of the deposition rate across the substrate surface. More importantly, these methods would change the diamond deposition chemistry from UNCD to MCD (microcrystalline diamond). MCD is significantly rougher than UNCD and is not a favorable option to improve the antithrombogenicity of the resultant surfaces [16]. Here we developed a technique to minimize the residual film stress. Briefly, the coated samples were exposed to hot hydrogen right after the diamond deposition steps, which caused the sample surface temperature to be approximately $50{ }^{\circ} \mathrm{C}$ higher than the deposition temperature. This was a relatively short (5-35 minutes compared to hours for the main deposition steps) process, as more $\mathrm{H}$ atoms decarburized the filament and quickly raises the temperature of the substrate, similar to the anneal process for other coating techniques, but not in inert gases. In addition, in such a short time without involving carbon-related gases, the film thickness and grain size does did not change. Figure 2 demonstrates that 25 minutes of such treatment can achieve a stress close to $0 \mathrm{MPa}$, and can avoid the drawbacks caused by the 
aforementioned methods. When the stress is under $200 \mathrm{MPa}$, the curvature change of a leaflet is not measurable. Because of very small thickness and low stress introduced, the leaflets and orifices could be easily assembled without impacting the geometry of the valves.
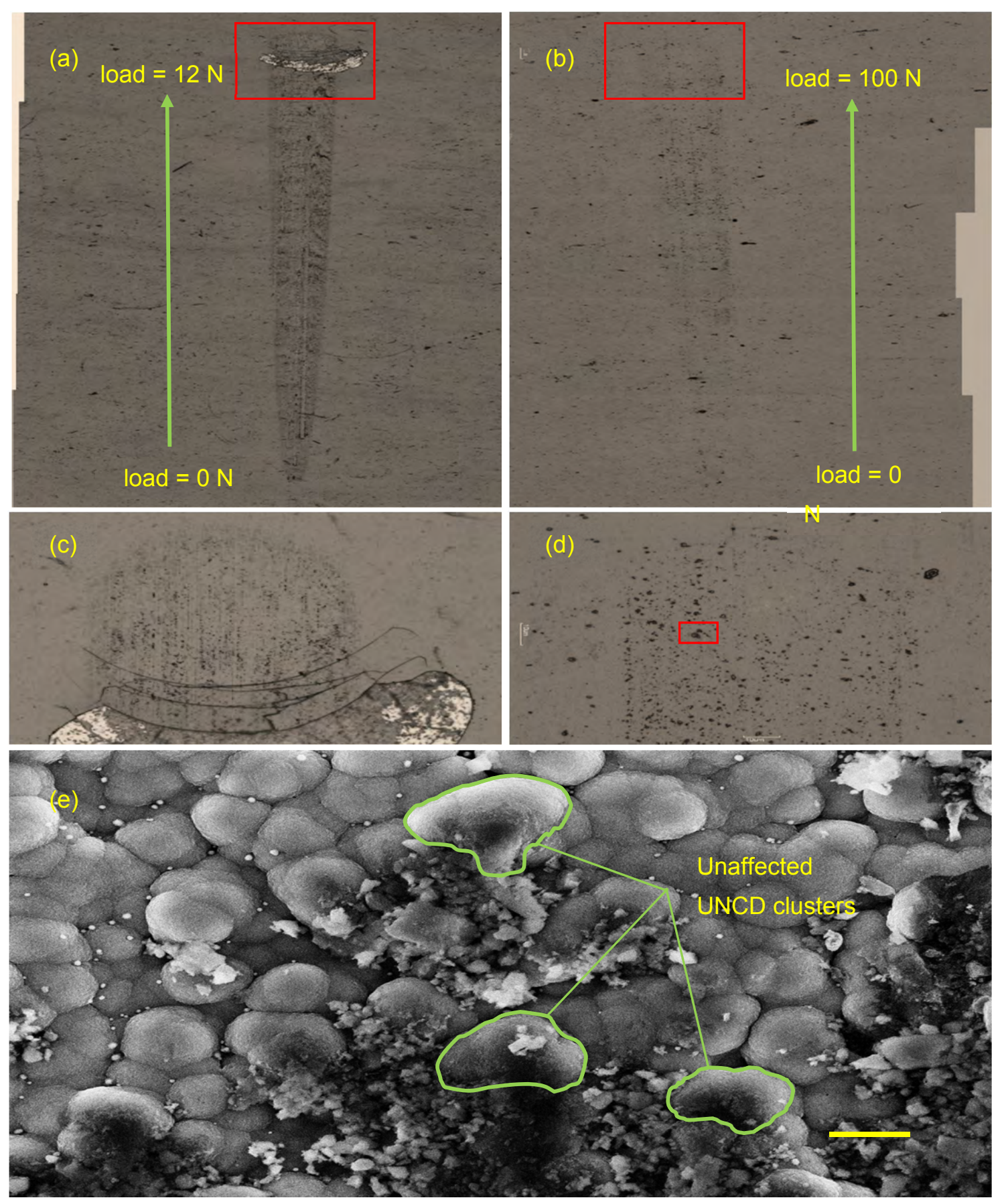

Fig 3. Scratch test on UNCD films deposited on PyC. a) with undeveloped technique at 12-N load inspected under an optical microscope with $50 \times$ magnification; b) with $200 \times$ magnification; c) with developed technique at $100-\mathrm{N}$ load under optical microscope with 50× magnification; d) with $200 \times$ magnification; e) SEM image of debris scraped from the Thomson Precision Ball of the scratch tester with a $2-\mu \mathrm{m}$ scale bar. Details in red squares in a), b) and d) are presented in c), d) and e) respectively, with higher magnification. 
A more thorough test on the adhesion of UNCD on PyC was conducted through a scratch test. Before adding the tungsten interlayer and the transit step, the UNCD film on PyC substrate would not survive a scratch test with a load higher than $10 \mathrm{~N}$. Figure $3 \mathrm{a}$ and $3 \mathrm{c}$ show that at the load of $12 \mathrm{~N}$, cracks appeared; however, with the developed UNCD-PyC integration technique, the film could survive a load up to $100 \mathrm{~N}$. Figure $3 \mathrm{~b}$ and $3 \mathrm{~d}$ demonstrate that even at the end of the scratch test, i.e., the load reached $100 \mathrm{~N}$, no crack was observed under $1000 \times$ magnification. The marks left by the Thomson Precision Ball on the undeveloped and the developed coating looked different. The mark that made of a series of small cracks on the undeveloped coating was visible under an optical microscope, and the cause of the mark on the developed coating was inspected under SEM as shown in Figure 3e. The SEM image reveals that the mark was not made of cracks but debris left by the ball material scraped by the UNCD surface. If the hardness and robustness of the ball material was equal to or higher than that of the UNCD, we would be able to see cracks and detachment of the UNCD clusters as marked in Figure 3e. However, no UNCD cluster broken or detached from the substrate (in pebble shape) was observed during the scratch test. When the PyC substrate was not coated with UNCD, the Thomson Precision Ball with ceramic material could readily break into the substrate. These results suggest that, after coating a few micron of UNCD, the hardness of the PyC was significantly elevated. The contact area between the ceramic ball and the sample was a circle with a diameter of approximately $200 \mu \mathrm{m}$ at the end of

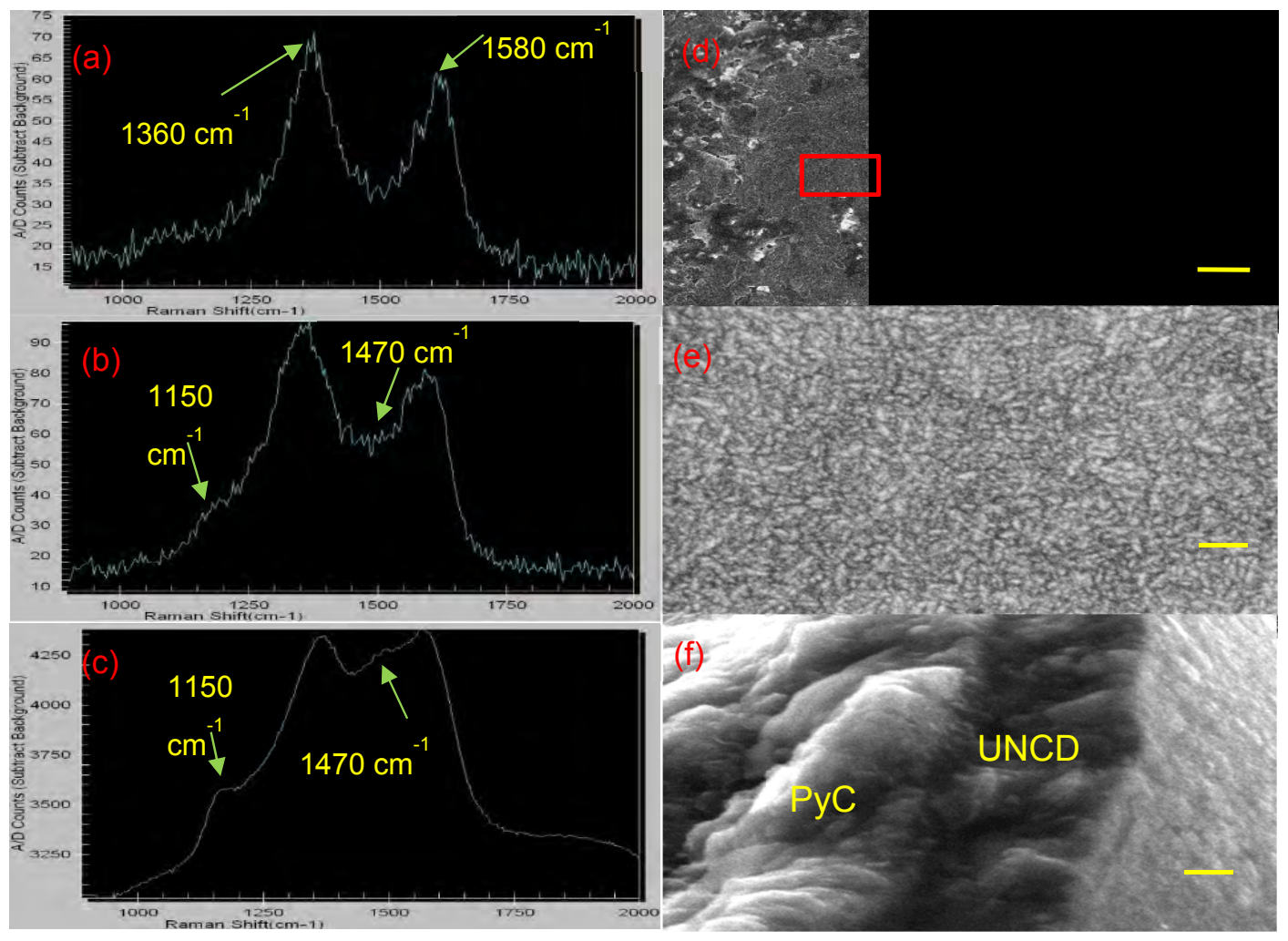

Fig 4. Raman spectrum of a) PyC, b) transit layer, and c) UNCD on top layer, d) SEM image of UNCD-coated PyC with 2- $\mu \mathrm{m}$ scale bar and a magnified square in red showing e) details of UNCD coating with a 100-nm scale bar, f) cross section SEM image of UNCD on PyC substrate with a 1- $\mu$ m scale bar. 
the test. With the maximum load of $100 \mathrm{~N}$, it was easy to calculate that the pressure applied on the film was about $3 \times 10^{9} \mathrm{~Pa}$.

Raman characterization of the samples was rigorously done during the whole deposition development. As described in the experimental section, we first studied the Raman signature of the bare PyC (Figure 4a) which only contained the so-called D-band and G-band at $1360 \mathrm{~cm}^{-1}$ and $1580 \mathrm{~cm}^{-1}$ [13]. At the beginning of the diamond deposition, a transit deposition step was introduced to bridge the coating from the PyC form to eventually the UNCD form. At the end of this step, a second Raman spectrum was measured, as shown in Figure $4 \mathrm{~b}$. Besides the PyC signatures, the spectrum contained signatures of nanocrystalline diamond at 1150 $\mathrm{cm}^{-1}$ and $1470 \mathrm{~cm}^{-1}$, indicating the presence of hydrocarbon [14], more specifically, transpolyacetylene lying in grain boundaries of NCD or UNCD [15]. The Raman spectra suggested that, during the transit step, more NCD or UNCD had been formed. We understand that the tungsten interlayer effectively prevented PyC from absorbing the precursors $\mathrm{CH}_{\mathrm{x}}(\mathrm{x}=1,2$ or 3$)$ in the reactor, therefore these saved precursors were used to form diamond. The interlayer also reacted with the thermal carbon species to form a strong tungsten carbide bond with the diamond nucleation centers. After the transit step, the film deposition conditions were fully changed to grow pure UNCD. Figure $4 c$ depicts a typical Raman spectrum of UNCD with $\mathrm{sp}^{2}$ impurity $<4 \%$ reported in previous work [9]. The top view SEM image (Figure 4e) confirms a typical high purity UNCD film aspect, characterized by faceted grains and clusters. The transit layer may also have compensated the mismatch of coefficient of thermal expansion (CTE) between the PyC substrate $\left(\sim 5 \times 10^{-6} \mathrm{~K}^{-1}\right)$ and the UNCD film $\left(1 \times 10^{-6}\right.$ $\mathrm{K}^{-1}$ ), and provided a stress buffer between them, however, because its continuous structural transition from PyC to UsNCD, this transition layer was too thin to be distinguished in the SEM cross section image in Figure 4f.

Using a standard prothromobinase assay, thrombin generation of fresh human platelets was measured after platelets were exposed to UNCD, PyC, MCD (microcrystalline diamond) and Silicon Carbide (SiC), which were all reported as good biocompatible materials candidates. The results indicated that most of these materials had acceptable biocompatibility towards platelets. Figure 5 demonstrates that thrombin generation induced by UNCD was slightly lower than that induced by PyC, but no significant difference was detected between the two conditions. Thrombin generation induced by these two materials was very similar to that of control platelets, i.e., almost "invisible" to the interacted fresh human platelets. However, $\operatorname{MCD}(P=0.015)$ and $\mathrm{SiC}(P=0.006)$ induced a significant increase in thrombin generation compared to control platelets. Platelets treated with calcium ionohpore A23187 was used as the positive control (i.e., maximum platelet activation and thrombin generation). Thrombin generation from ionophore treated platelets was approximately $300 \%$ higher than that of the negative control, therefore data obtained from the positive control was not shown in Figure 5. These results are compatible with our previous experiment of fibrinogen adsorption on UNCD surface versus surface of other biocompatible materials [16]. It is noted that boron doped UNCD (BD-UNCD) induced a similar level of thrombin generation compared to PyC, suggesting that electronically active devices made of BD-UNCD, such as sensors or energy collectors could become 
implantable. More systematic experiments are being conducted currently to evaluate the biocompatibility and hemodynamic performance of UNCD-coated heart valves.

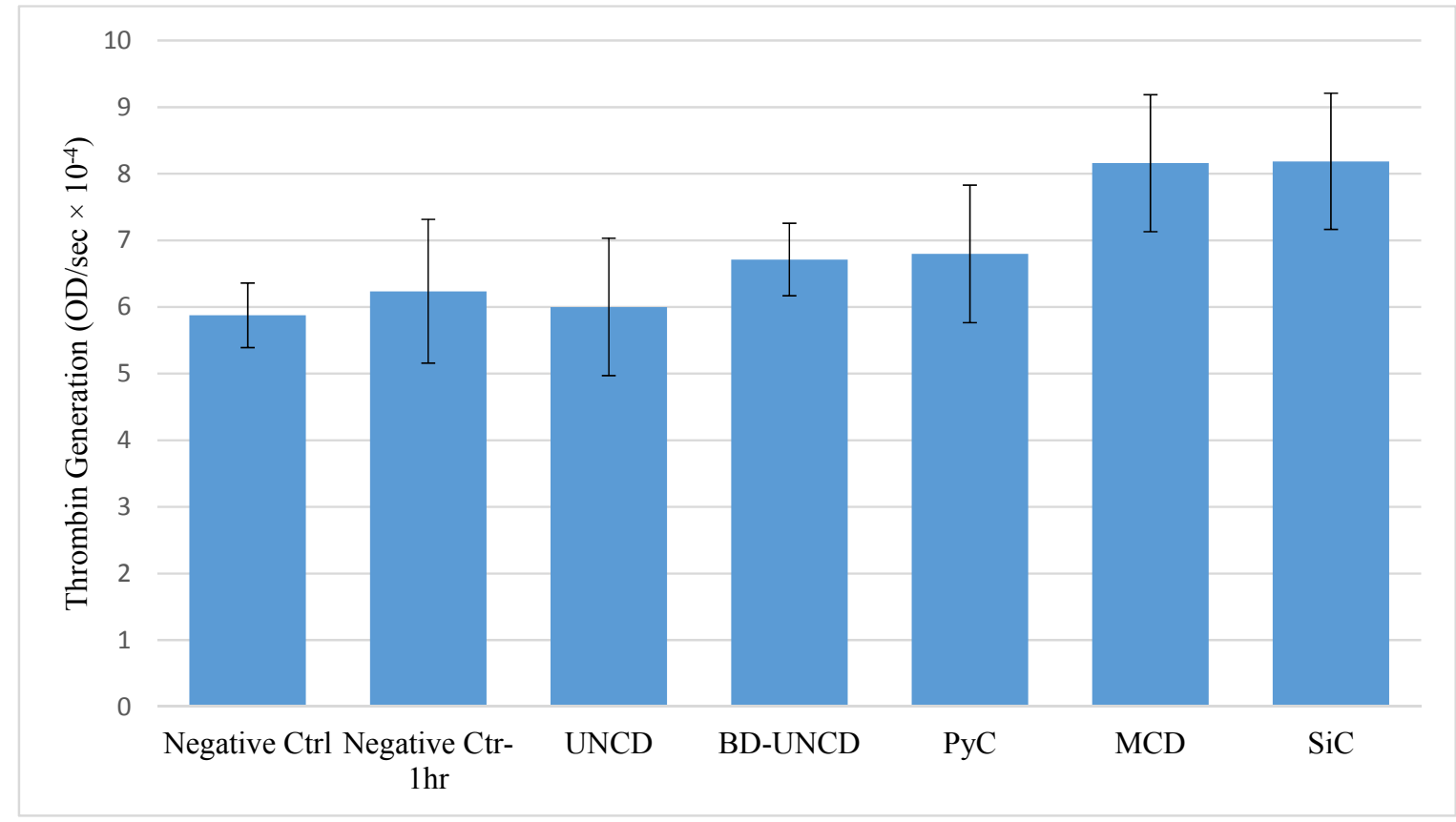

Fig 5. Thrombin generation of fresh human platelets after1-hour exposure to UNCD, BD-UNCD, PyC, and MCD. Platelets without any treatment was used as the negative control.

\section{Conclusions}

We have demonstrated a strong integration between a UNCD film and PyC heart valve components. The strong integration has been achieved by combining a very conformal tungsten interlayer technique and a transit step to enforce carbide bonding at UNCD-PyC interface, which minimized graphitization as well as film stress, and compensated CTE mismatch between PyC and UNCD. Such development ensured a high UNCD quality and strong film adhesion on the PyC substrate. The coating had minimum impact on the geometry of the component of the PyC heart valve. An aggressive scratch test showed that the UNCD film on PyC leaflets can survive a load of $100 \mathrm{~N}$ on a contact area of $3 \times 10^{-2} \mathrm{~mm}^{2}$, free of any sign of film damage (crack or delamination). Contact with UNCD induced a control level thrombin generation in human platelets, which was comparable or less than PyC, and significantly less than MCD and SiC. In short, UNCD coating could significantly improve the $\mathrm{PyC}$ valves' mechanical performance without sacrificing the biocompatibility of the valves. Techniques and methods reported in this study could surely benefit any other PyC-based implantable devices as well.

\section{Acknowledgments}

The work was supported through a Phase II SBIR grant from NIH, R44HL108534. Heart valves were provided by Meril Life Sciences Pvt. Ltd. SEM and optical profilometry use of the Center for Nanoscale Materials (CNM), Argonne National Laboratory were supported by the U. S. Department of Energy, Office 
of Science, Office of Basic Energy Sciences, under Contract No. DE-AC02-06CH11357. The authors would like to thank Leonidas Ocola, Ralu Divan, and Liliana Stan at CNM for their valuable help and discussion on SEM imaging, optical profilometry and atomic layer deposition. The authors thank Keith Gantz, Jack Lusk and Dale McClure at ADT for diamond film characterizations.

\section{References}

[1] V. L.Gott, D. E.Alejo, and D. E. Cameron, Mechanical Heart Valves: 50 Years of Evolution, Ann. Thorac. Surg. 76 (2003) S2230.

[2] Hongbo Zhang, Xiaoyan Deng, Tomas Francisco Cianciulli, Ze Zhang, Daniel Chappard, Jorge Alberto Lax, Maria Cristina Saccheri, Hector Jorge Redruello, Jorge Luis Jordana, Horacio Alberto Prezioso, Martin King, Robert Guidoin, Clinical Device-Related Article Pivoting System Fracture in a Bileaflet Mechanical Valve: A Case Report, J. Biomedical Materials Research Part B: Applied Biomaterials 90B (2009) 952.

[3] A. Grill, Diamond-like carbon coatings as biocompatible materials - an overview, Diam. Relat. Mater. 12, $166(2003) 166$

[4] E. Baudet, V. Puel, J. McBride, J. P Grimaud, F. Roques, F. Clerc, X. Roques, N. Laborde, Surgery for acquired heart disease, J. of Thoracic and Cardiovascular Surgery 109(1995) 858

[5] A.R. Krauss, O. Auciello, D.M. Gruen, A. Jayatissa, A. Sumant, J. Tucek, D.C. Mancini, N. Moldovan, A. Erdemir, D. Ersoy, M.N. Gardos, H.G. Busmann, E.M. Meyer, M.Q. Ding, Ultrananocrystalline diamond thin films for MEMS and moving mechanical assembly devices, J. Appl. Phys. 89 (2001) 2958

[6] Piyush Bajaj, Demir Akin, Amit Gupta, Debby Sherman, Bing Shi, Orlando Auciello, Rashid Bashir, Ultrananocrystalline diamond film as an optimal cell interface for biomedical applications, Biomed Microdevices 9 (2007) 787.

[7] J. C. Bokros, The structure of pyrolytic carbon deposited in a fluidized bed, Carbon 3(1965) 17.

[8] J.E., Butler, A. V. Sumant, The CVD of Nanodiamond Materials, Chem Vap Deposition 14(2008) 145

[9] Hongjun Zeng, Andrew R. Konicek, Nicolaie Moldovan, Filippo Mangolini, Tevis Jacobs, Ian Wylie, Prabhu U. Arumugam, Shabnam Siddiqui, Robert W. Carpick, John A. Carlisle, Boron-doped ultrananocrystalline diamond synthesized with an H-rich/Ar-lean gas system, CARBON 84(2015) 103.

[10] R. Shi, H. J. Li, Z. Yang, and M. K. Kang, Deposition mechanism of pyrolytic carbons at temperature between $800-1200^{\circ} \mathrm{C}$, Carbon 35(1997) 1789.

[11] V. De Pauw, S. Kalhöfer, and D. Gerthsen, Influence of the deposition parameters on the texture of pyrolytic carbon layers deposited on planar substrates, Carbon, 42(2004) 279.

[12] W. Yin and D. Rubenstein, Dose Effect of Shear Stress on Platelet Complement Activation in a Cone and Plate Shearing Device, Cellular and Molecular Bioengineering, 2(2009) 274.

[13] E. López-Honorato, P. J. Meadows, R. A. Shatwell and P. Xiao, Characterization of the anisotropy of pyrolytic carbon by Raman spectroscopy, Carbon 48 (2010)881. 
[14] W. Gajewski, P. Achatz, O.A. Williams, K. Haenen, E. Bustarret, M. Stutzmann and J.A. Garrido, Electronic and optical properties of boron-doped nanocrystalline diamond films, Physics Review B, 79 (2009) 045206.

[15] A. C. Ferrari and J. Robertson, Origin of the 1150-cm-1 Raman mode in nanocrystalline diamond, Physics Review B, 63(2001) 121405(R).

[16] H. Zeng, P. U. Arumugam, and J. A. Carlisle, Fibrinogen adsorption study on ultrananocrystalline diamond as a biocompatible and antithrombogenic interfacial material for implantable devices, Phys. Status Solidi A 211(2014) 2785 
